This item was submitted to Loughborough's Research Repository by the author.

Items in Figshare are protected by copyright, with all rights reserved, unless otherwise indicated.

\title{
Eigenvalue estimates for bilayer graphene
}

PLEASE CITE THE PUBLISHED VERSION

https://doi.org/10.1007/s00023-019-00770-x

PUBLISHER

Springer

VERSION

AM (Accepted Manuscript)

PUBLISHER STATEMENT

This is a post-peer-review, pre-copyedit version of an article published in Annales Henri Poincare. The final authenticated version is available online at: https://doi.org/10.1007/s00023-019-00770-x

\section{LICENCE}

CC BY-NC-ND 4.0

\section{REPOSITORY RECORD}

Cuenin, Jean-Claude. 2019. "Eigenvalue Estimates for Bilayer Graphene”. Loughborough University. https://hdl.handle.net/2134/13227638.v1. 


\title{
Eigenvalue estimates for bilayer graphene
}

\author{
Jean-Claude Cuenin
}

\begin{abstract}
Recently, Ferrulli-Laptev-Safronov [6] obtained eigenvalue estimates for an operator associated to bilayer graphene in terms of $L^{q}$ norms of the (possibly non-selfadjoint) potential. They proved that for $1<q<4 / 3$ all non-embedded eigenvalues lie near the edges of the spectrum of the free operator. In this note we prove this for the larger range $1 \leq q \leq 3 / 2$. The latter is optimal if embedded eigenvalues are also considered. We prove similar estimates for a modified bilayer operator with so-called "trigonal warping" term. Here, the range for $q$ is smaller since the Fermi surface has less curvature. The main tool are new uniform resolvent estimates that may be of independent interest and are collected in an appendix (in greater generality than needed).
\end{abstract}

\section{Introduction and main results}

Graphene is a two-dimensional material consisting of a single layer of carbon atoms arranged in a honeycomb lattice. Behind its many remarkable properties is the fact that at low-energy the dynamics of charge carriers in graphene is governed by the Dirac equation. Bilayer graphene is produced by stacking one layer on top of another, with a 60 degrees angle of rotation between the two layers. The effective Hamiltonian of bilayer graphene, in suitable units, is given by

$$
D_{m}=\left(\begin{array}{cc}
m & 4 \partial_{\bar{z}}^{2} \\
4 \partial_{z}^{2} & -m
\end{array}\right)
$$

where

$$
\partial_{\bar{z}}=\frac{1}{2}\left(\frac{\partial}{\partial x_{1}}+\frac{1}{i} \frac{\partial}{\partial x_{2}}\right), \quad \partial_{z}=\frac{1}{2}\left(\frac{\partial}{\partial x_{1}}-\frac{1}{i} \frac{\partial}{\partial x_{2}}\right),
$$

see e.g. formula (1.46) in [15] and the original references mentioned there. The parameter $m \geq 0$ plays the role of a mass term, similar as for the Dirac operator. However, it should not be confused with the physical mass (denoted by $m^{*}$ in [15]) of the charge carrier (i.e. the electron). Experimentally, a mass gap can be created by physical strain, see Vozmediano et al [22].

We will consider the operator $D:=D_{m}+V$ on the Hilbert space $L^{2}\left(\mathbb{R}^{2} ; \mathbb{C}^{2}\right)$. The domain of $D$ is the Sobolev space $H^{2}\left(\mathbb{R}^{2} ; \mathbb{C}^{2}\right)$. The potential 
$V: \mathbb{R}^{2} \rightarrow \operatorname{Mat}(2 \times 2 ; \mathbb{C})$ is not assumed to take values in the self-adjoint matrices, i.e $D$ is allowed to be non-selfadjoint. From the point of view of physics an important motivation to consider non-self-adjoint operators comes from the study of resonances, either via the complex scaling method introduced by Aguilar-Combes [1] or via the method of complex absorbing potentials (see e.g Riss-Meyer [19] or Zworski [23].)

The spectrum of $D_{m}$ is absolutely continuous and given by

$$
\sigma\left(D_{m}\right)=(-\infty, m] \cup[m, \infty) .
$$

Note in particular that $\sigma\left(D_{0}\right)=\mathbb{R}$.

Laptev-Ferrulli-Safronov [6] proved the following eigenvalue estimate: If $V \in L^{q}\left(\mathbb{R}^{2}, \operatorname{Mat}(2 \times 2 ; \mathbb{C})\right)$ with $1<q<4 / 3$ and if $z \in \sigma_{\mathrm{p}}(D) \backslash \sigma\left(D_{m}\right)$, then

$$
C_{q}\|V\|_{q}^{q}\left|z^{2}-m^{2}\right|^{(1-q) / 2}\left(\sqrt{\left|\frac{z-m}{z+m}\right|}+\sqrt{\left|\frac{z+m}{z-m}\right|}+1\right)^{q} \geq 1 .
$$

Here, $C_{q}>0$ is independent of $V, z$ and $m$, and $\sigma_{\mathrm{p}}(D)$ denotes the point spectrum (the set of eigenvalues) of $D$. Our first result is that (1.1) is valid for the larger range $1 \leq q \leq 3 / 2$ and for any $z \in \sigma_{\mathrm{p}}(D)$. In view of the counterexamples in [3] this range is optimal if embedded eigenvalues are taken into account. Whether it is optimal for $z \in \sigma_{\mathrm{p}}(D) \backslash \sigma\left(D_{m}\right)$ is related to the Laptev-Safronov conjecture for Schrödinger operators [17]. We refer to [7, 10, 8] for a full discussion and progress towards a resolution of the conjecture.

We adopt the following notation, in line with [4], [5].

$$
\begin{aligned}
k(z)^{4} & :=z^{2}-m^{2}, \quad \arg (k(z)) \in[0, \pi / 2), \\
\zeta(z) & :=\frac{z+m}{k(z)^{2}}, \quad z \neq \pm m .
\end{aligned}
$$

Observe that $\zeta^{2}$ extends to a holomorphic map on the Riemann sphere $\mathbb{C} \cup$ $\{\infty\}$, with $\zeta^{2}(m)=\infty$ and $\zeta^{2}(-m)=0$. We abbreviate

$$
\|V\|_{q}^{q}:=\int_{\mathbb{R}^{2}}\|V(x)\|^{q} \mathrm{~d} x, \quad 1 \leq q<\infty,
$$

Here, $\|V(x)\|$ is the operator norm of $V(x) \in \operatorname{Mat}(2 \times 2 ; \mathbb{C})$ as a linear map on $\mathbb{C}^{2}$, when the latter is equipped with the Euclidean norm.

Theorem 1.1. Let $V \in L^{q}\left(\mathbb{R}^{2}, \operatorname{Mat}(2 \times 2 ; \mathbb{C})\right)$, with $1 \leq q \leq 3 / 2$. Then the following estimates hold for $D:=D_{m}+V$ with some constant $C_{q}>0$ independent of $V, z$ and $m$.

(i) If $1<q \leq 3 / 2$, then every $z \in \sigma_{\mathrm{p}}(D)$ satisfies

$$
\frac{|k(z)|^{2 q-2}}{\left(1+|\zeta(z)|+|\zeta(z)|^{-1}\right)^{q}} \leq C_{q}\|V\|_{q}^{q} .
$$

In particular, if $m=0$, the left hand side is proportional to $|z|^{q-1}$.

(ii) If $q=1$, then there exists $c_{1}>0$ such that if $\|V\|_{1} \leq c_{1}$, then

$$
\sigma_{\mathrm{p}}(D) \subset\left\{z \in \mathbb{C}:|z \pm m| \leq C_{1} m\|V\|_{1}^{2}\right\} \quad \text { if } m>0
$$


whereas for $m=0$ we have $\sigma_{\mathrm{p}}(D)=\emptyset .^{1}$

Remark 1.2. The range $1<q \leq 3 / 2$ in Theorem 1.1 is the same as for the Schrödinger operator $-\Delta+V$ in two dimensions, with $V \in L^{q}\left(\mathbb{R}^{2}\right)$, for which the inequality

$$
|z|^{q-1} \leq C_{q}\|V\|_{q}^{q}
$$

was proved by Frank [7]. This is no coincidence since $D_{m}$ and $-\Delta$ are both second order differential operators. Note, however, that the analogue of (1.5), formally corresponding to (1.6) with $q=1$, is false, even for real-valued potentials. Indeed, it is well known that for $V \leq 0$ and not identically zero, $-\Delta+V$ always has a negative eigenvalue. The failure of the $q=1$ bound stems from the logarithmic singularity of the fundamental solution of $-\Delta$. In contrast, the fundamental solution of $D_{m}$ is bounded, see (2.5). A more intuitive way to see this is to observe that the fundamental solution of $\partial_{z}^{2}$, given by $z / \bar{z}$, is bounded.

Our second result deals with a modified version of the operator $D_{m}$; we consider the case $m=0$ for simplicity, but the case $m>0$ could be treated along the same lines. Recall that $D_{0}$ is an effective low-energy Hamiltonian for bilayer graphene. It arises as the leading order approximation of a tightbinding Hamiltonian, see e.g [15]. If one takes higher order terms (largerdistance hopping processes) into account the effective Hamiltonian is modified to

$$
D_{\text {trig }}=\left(\begin{array}{cc}
0 & 4 \partial_{\bar{z}}^{2}+2 \alpha \partial_{z} \\
4 \partial_{z}^{2}+2 \alpha \partial_{\bar{z}} & 0
\end{array}\right),
$$

where $\alpha>0$ depends on the physical paramters of the model, see (1.53) in [15]. We will set $\alpha=1$ for simplicity; its original value can easily be restored by dimensional analysis. The eigenvalues $\lambda_{ \pm}(\xi)$ of the symbol of $D_{\text {trig }}$ are given by

$$
\lambda_{ \pm}(\xi):= \pm \sqrt{P(\xi)}, \quad \text { where } P(\xi):=|\xi|^{4}+2 \operatorname{Re}\left(\xi^{3}\right)+|\xi|^{2} .
$$

The cubic term can be written in polar coordinates $\xi=|\xi| \mathrm{e}^{\mathrm{i} \theta}$ as $2|\xi|^{3} \cos (3 \theta)$. Therefore, the dispersion relations $\lambda_{ \pm}(\cdot)$ are only invariant under 120 degree rotations. This effect is called "trigonal warping" in the physical literature and explains the subscript "trig".

Theorem 1.3. Let $V \in L^{q}\left(\mathbb{R}^{2}, \operatorname{Mat}(2 \times 2 ; \mathbb{C})\right)$, with $1 \leq q \leq 3 / 2$. Then the following estimates hold for $D:=D_{\text {trig }}+V$ with some constant $C_{q}>0$ independent of $V$ and $z$.

(i) We have the inclusion

$$
\sigma_{\mathrm{p}}(D) \subset\left\{z \in \mathbb{C}:|z|^{q-1} \leq C_{q}\left(1+\|V\|_{q}^{q}\right)\right\}
$$

\footnotetext{
${ }^{1}$ In fact, if $m=0$ and $\|V\|_{1}$ is sufficiently small, then $D$ and $D_{0}$ are similar. This follows from Kato's theory of smooth perturbations [14, Theorem 1.5] and the fact that (2.2) is uniformly bounded for $m=0$ and $q=1$.
} 
(ii) There exists $c>0$ such that if $V \in\left(L^{q} \cap L^{q_{0}}\right)\left(\mathbb{R}^{2}, \operatorname{Mat}(2 \times 2 ; \mathbb{C})\right)$, where $1<q \leq 3 / 2$ and $q_{0}=\min (q, 5 / 4)$, and if $\|V\|_{q_{0}} \leq c$, then

$$
\sigma_{\mathrm{p}}(D) \subset\left\{z \in \mathbb{C}:|z|^{q-1} \leq C_{q}\|V\|_{q}^{q}\right\} \cup\left\{z \in \mathbb{C}:|z-1 / 16|^{q-1} \leq C_{q}\|V\|_{q}^{q}\right\} .
$$

(iii) If $q=1$, then there exists $c_{1}>0$ such that if $0<\|V\|_{1} \leq c_{1}$, then

$$
\sigma_{\mathrm{p}}(D) \subset\left\{z \in \mathbb{C}:|z-1 / 16| \leq \exp \left(-C_{1}\|V\|_{1}^{-1}\right)\right\} .
$$

Remark 1.4. In view of the counterexamples in [3], (i) is optimal in the sense that (1.8) cannot hold for any $q>3 / 2$. The situation regarding (ii), (iii) is less clear. Remark (2.7) shows that there exist $\lambda>0$ for which the Fermi surface $M_{\lambda}=\left\{\xi \in \mathbb{R}^{2}: P(\xi)=\lambda\right\}$ has points of vanishing curvature. Theorem 1.2 in [3] then shows that (1.9) cannot hold for $q>4 / 3$ if the assumption $V \in L^{5 / 4}\left(\mathbb{R}^{2}, \operatorname{Mat}(2 \times 2 ; \mathbb{C})\right)$ is dropped. An open problem is thus whether (1.9) is true under the assumption that $V \in L^{q}\left(\mathbb{R}^{2}, \operatorname{Mat}(2 \times 2 ; \mathbb{C})\right)$, with $5 / 4<q \leq 4 / 3$. Concerning (iii), another open problem is whether the stronger conclusion $\sigma_{\mathrm{p}}(D)=\emptyset$ holds provided $c_{1}$ is sufficiently small. The obstruction in our proof is the logarithmic loss in the constant of Proposition A.11.

The outline of this note is as follows. In section 2 we prove uniform resolvent estimates in Schatten spaces for the bilayer operators $D_{m}$ and $D_{\text {trig. }}$. These follow from the more general results in the appendix. In Section 3 we prove Theorems 1.1-1.3.

Even though operator norm estimates would be sufficient for our purpose here, we state our resolvent estimates with the stronger Schatten norm bounds for future reference. Such bounds were pioneered by Frank-Sabin [9] for the Laplacian.

\section{Resolvent estimates}

\subsection{Fourier transforms of arclength measures}

Definition 2.1. A smooth curve $C \subset \mathbb{R}^{2}$ is called of finite type at $\xi_{0} \in C$ if its curvature $\kappa$ does not vanish to infinite order at $\xi_{0}$. The smallest $k \in \mathbb{N}_{\geq 2}$ such that $\kappa^{(k-2)}\left(\xi_{0}\right) \neq 0$ is called the type of $C$ at $\xi_{0}$. For any compact subset $C^{\prime} \subset C$, the type of $C^{\prime}$ is defined as the maximum of the types of $\xi_{0} \in C^{\prime}$.

Remark 2.2. Suppose that, after translation and rotation, $\xi_{0}=0$ and $C$ is locally the graph of a smooth map $h: U \rightarrow \mathbb{R}^{2}$ where $U$ is a neighborhood of the origin in $\mathbb{R}$ and $h(0)=h^{\prime}(0)=0$. Then

$$
\kappa=\frac{h^{\prime \prime}}{\left(1+\left(h^{\prime}\right)^{2}\right)^{3 / 2}} .
$$

Hence the type of $C$ at the origin equals the smallest $k \in \mathbb{N}_{\geq 2}$ such that $h^{(k)}(0) \neq 0$. 
Lemma 2.3. Let $C \subset \mathbb{R}^{2}$ be a smooth compact curve of type $k$. Then the Fourier transform of the arclength measure on $C$ is $\mathcal{O}\left((1+|x|)^{-1 / k}\right)$.

Proof. This follows from van der Corput's lemma, see e.g. Proposition 2 (and its corollary) in Section VIII of [21].

\subsection{Resolvent estimates for $D_{m}$}

In the following, $\alpha_{q, r, d} \in(1, \infty)$ is as defined in (A.2).

Proposition 2.4. Let $1 \leq q \leq 3 / 2$. There exists $C>0$ such that for any $A, B \in L^{2 q}\left(\mathbb{R}^{2}, \operatorname{Mat}(2 \times 2 ; \mathbb{C})\right)$ and $z \in \rho\left(D_{m}\right)$ we have the inequality

$$
\left\|A\left(D_{m}-z\right)^{-1} B\right\|_{\mathfrak{S}^{\max \left(q, \alpha_{q, 1 / 2,2}\right)}} \leq C|k(z)|^{\frac{2}{q}-2}\left(|\zeta(z)|+|\zeta(z)|^{-1}\right)\|A\|_{2 q}\|B\|_{2 q} .
$$

Proof. By scaling, we may assume that $|k(z)|=1$. By a limiting argument, we may also assume that $k(z) \neq 1$, so $k(z)=\mathrm{e}^{\mathrm{i} \varphi}$ with $\varphi \in(0, \pi / 2)$. Since $D_{m}^{2}=\left(\Delta^{2}+m^{2}\right) \mathbf{1}_{2 \times 2}$, we have the identity

$$
\left(D_{m}-z\right)^{-1}=\left(D_{m}+z\right)\left(\Delta^{2}-k(z)^{4}\right)^{-1} .
$$

It is therefore enough to prove the estimate $(2.2)$ with $\left(D_{m}-z\right)^{-1}$ replaced by the Fourier multipliers $m_{j}(-\mathrm{i} \nabla), j=1,2$, with

$$
m_{1}(\xi):=\frac{1}{|\xi|^{4}-\mathrm{e}^{\mathrm{i} \varphi}}, \quad m_{2}(\xi):=\frac{\left(\xi_{1} \pm \mathrm{i} \xi_{2}\right)^{2}}{|\xi|^{4}-\mathrm{e}^{\mathrm{i} \varphi}} .
$$

Let $\chi_{1} \in C_{c}^{\infty}\left(\mathbb{R}^{2} ;[0,1]\right)$ be supported on $\left\{\xi \in \mathbb{R}^{2}: 1 / 2 \leq|\xi| \leq 3 / 2\right\}$ and equal to 1 on $\left\{\xi \in \mathbb{R}^{2}: 3 / 4 \leq|\xi| \leq 5 / 4\right\}$, and set $\chi_{2}:=1-\chi_{1}$. Then it remains to prove the estimates

$$
\left\|A \chi_{i}(-\mathrm{i} \nabla) m_{j}(-\mathrm{i} \nabla) B\right\|_{\mathfrak{S}^{\alpha_{q}}} \leq C\|A\|_{2 q}\|B\|_{2 q}, \quad i, j=1,2,
$$

with $C$ independent of $\varphi$. Since the zero set of $|\xi|^{4}-1$ is the unit circle the case $i=1$ follows from Corollary A.9 or A.8. ${ }^{2}$ The case $i=2$ and $1<q \leq 3 / 2$ follows from the Kato-Seiler-Simon inequality [20, Theorem 4.1] and Hölder's inequality in Schatten spaces. In fact, for $q>1$, we have

$$
\left\|A \chi_{2}(-\mathrm{i} \nabla) m_{j}(-\mathrm{i} \nabla) B\right\|_{\mathfrak{S}^{q}} \leq C\left\|\left(|\cdot|^{2}+1\right)^{-1}\right\|_{q}\|A\|_{2 q}\|B\|_{2 q},
$$

which is again better than the claimed bound. The case $j=2$ and $q=1$ would follow from the estimate

$$
\sup _{x \in \mathbb{R}^{2} \backslash\{0\}}\left|\int_{\mathbb{R}^{2}} \mathrm{e}^{\mathrm{i} x \cdot \xi} \frac{\left(\xi_{1} \pm \mathrm{i} \xi_{2}\right)^{2}}{|\xi|^{4}+1} \mathrm{~d} \xi\right|<\infty
$$

since the latter implies boundedness of the kernel of $\chi_{2}(-\mathrm{i} \nabla) m_{2}(-\mathrm{i} \nabla)$ and hence the Hilbert-Schmidt bound

$$
\left\|A \chi_{2}(-\mathrm{i} \nabla) m_{2}(-\mathrm{i} \nabla) B\right\|_{\mathfrak{S}^{2}} \leq C\|A\|_{2 q}\|B\|_{2 q} .
$$

${ }^{2}$ In fact, since $2\left(|\xi|^{4}-k^{4}\right)^{-1}=\left(|\xi|^{2}-k^{2}\right)^{-1}-\left(|\xi|^{2}+k^{2}\right)^{-1}$ it would also follow from Kenig-Ruiz-Sogge [16]. 
To prove (2.5) fix $x \in \mathbb{R}^{2} \backslash\{0\}$ and choose polar coordinates $\xi=r \mathrm{e}^{\mathrm{i} \theta}$ where $\theta$ is the angle between $\xi$ and $x$. The integral in (2.5) then becomes

$$
\int_{0}^{\infty}\left(\int_{0}^{2 \pi} \mathrm{e}^{\mathrm{i} r|x| \cos (\theta)} \mathrm{e}^{2 \mathrm{i} \theta} \mathrm{d} \theta\right) \frac{r^{3}}{r^{4}+1} \mathrm{~d} r .
$$

Integration by parts shows that the $\theta$-integral is $\mathcal{O}(r|x|)$ for $r|x| \leq 1$. Stationary phase estimates yield an $\mathcal{O}\left((r|x|)^{-1 / 2}\right)$ bound for $r|x|>1$. Splitting the $r$-integral into a part where $r \leq|x|^{-1}$ and a part where $r>|x|^{-1}$ then yields $(2.5)$.

\subsection{Resolvent estimates for $D_{\text {trig }}$}

In the following we consider the symbol $P$, given by (1.7), as well as the curve (the "Fermi surface")

$$
M_{\lambda}=\left\{\xi \in \mathbb{R}^{2}: P(\xi)=\lambda\right\} .
$$

Lemma 2.5. The set of critical values of $P$ is $\{0,1 / 16\}$. The set of critical points is $\mathcal{C}=\mathcal{C}_{0} \cup \mathcal{C}_{1}$, where

$$
\begin{aligned}
& \mathcal{C}_{0}=P^{-1}(\{0\}) \cap \mathcal{C}=\left\{0, e^{\mathrm{i} \pi / 3}, e^{\mathrm{i} \pi}, e^{\mathrm{i} 5 \pi / 3}\right\}, \\
& \mathcal{C}_{1}=P^{-1}(\{1 / 16\}) \cap \mathcal{C}=\left\{e^{\mathrm{i} \pi / 3} / 2, e^{\mathrm{i} \pi} / 2, e^{\mathrm{i} 5 \pi / 3} / 2\right\} .
\end{aligned}
$$

All critical points are non-degenerate. In fact, the points in $\mathcal{C}_{0}$ are minima, and the points in $\mathcal{C}_{1}$ are saddle points. $M_{\lambda}$ is compact for all $\lambda \in \mathbb{R}_{+}$I $\{0,1 / 16\}$, has four connected components for $0<\lambda<1 / 16$ and is connected for $\lambda>1 / 16$.

Proof. In polar coordinates $\xi=r \mathrm{e}^{\mathrm{i} \theta}$ we have

$$
\widetilde{P}(r, \theta):=P\left(r \mathrm{e}^{\mathrm{i} \theta}\right)=r^{4}+2 r^{3} \cos (3 \theta)+r^{2}
$$

The $(r, \theta)$-gradient is

$$
\nabla_{(r, \theta)} \widetilde{P}(r, \theta)=\left(4 r^{3}+6 r^{2} \cos (3 \theta)+2 r\right) e_{r}+\left(-6 r^{2} \sin (3 \theta)\right) e_{\theta} .
$$

It vanishes whenever

$$
r=0, \quad \text { or } \quad\left(\sin (3 \theta)=0 \text { and } 4 r^{3}-6 r^{2}+2 r=0\right),
$$

where we used that $\cos (3 \theta)= \pm 1$ whenever $\sin (3 \theta)=0$, and that the equation $\nabla_{(r, \theta)} \widetilde{P}(r, \theta)=0$ with $\cos (3 \theta)=1$ does not have a solution $r>0$. The angles where $\cos (3 \theta)=-1$ are $\theta_{j}=\pi(1+2 j) / 3, j=0,1,2$. The nonzero solutions of the rightmost equation in (2.7) are $r=1$ and $r=1 / 2$. One easily checks that the critical values are 0 and $1 / 16$ and that the Hessian is nondegenerate at the critical points. Compactness of $M_{\lambda}$ follows from the fact that $P(\xi) \rightarrow+\infty$ as $|\xi| \rightarrow+\infty$. The claim about the number of connected components follows from Morse theory.

Lemma 2.6. For any $\lambda \in \mathbb{R} \backslash\{0,1 / 16\}$ the curve $M_{\lambda}$ is of finite type $k=4$. 
Proof. After rotation and translation we may assume that $M_{\lambda}$ is locally a graph of a smooth function $h: U \rightarrow \mathbb{R}$ over the $\xi_{2}$ axis such that $h(0)=$ $h^{\prime}(0)=0$. By Remark 2.2 it suffices to show that

$$
\sum_{k=2}^{4}\left|h^{(k)}(0)\right| \neq 0 \text {. }
$$

Assume the right hand side of (2.8) is zero. Repeatedly differentiating the identity $P\left(h\left(\xi_{2}\right), \xi_{2}\right)=\lambda$ at the point $\xi_{2}=0$ yields

$$
0=h^{(k)}(0)=-\frac{\partial_{\xi_{2}}^{(k)} P(0)}{\partial_{\xi_{1}} P(0)}, \quad k=1, \ldots, 4 .
$$

Since $P$ is a polynomial of degree four this implies that $t \mapsto P\left(t e_{2}\right)$ is constant. However, for any rotation matrix $R$ and for any $a \in \mathbb{R}^{2}$, the $t^{4}$-coefficient of the polynomial $t \mapsto P\left(t R e_{2}+a\right)$ is equal to 1 . Undoing the translation and rotation, we arrive at a contradiction.

Remark 2.7. It may be seen by explicit computation that $k=4$ is optimal in general. Indeed, changing variables to $\xi_{1}=\frac{1}{2}(3-\sqrt{7})+s, \xi_{2}=t$, one finds that

$$
P\left(\frac{1}{2}(3-\sqrt{7})+s, t\right)=\frac{233}{4}-22 \sqrt{7}+t^{4}+\left(72-27 \sqrt{7}+\mathcal{O}\left(t^{2}\right)\right) s+\mathcal{O}\left(s^{2}\right) .
$$

Then, for $\lambda:=\frac{233}{4}-22 \sqrt{7}$ and $n=1,2,3$, we have

$$
P\left(\frac{1}{2}(3-\sqrt{7}), 0\right)=\lambda, \quad \partial_{s} P\left(\frac{1}{2}(3-\sqrt{7}), 0\right) \neq 0, \quad \partial_{t}^{n} P\left(\frac{1}{2}(3-\sqrt{7}), 0\right)=0,
$$

and thus, by a parallel argument as that leading to (2.9), the curvature of $M_{\lambda}$ vanishes to second order at $\xi=\left(\frac{1}{2}(3-\sqrt{7}), 0\right)$.

We remind the reader that $\alpha_{q, r, d} \in(1, \infty)$ is defined in (A.2).

Proposition 2.8. Let $1 \leq q \leq 3 / 2$ and let $\mathcal{C}$ be given by (2.6). There exist $\delta_{1}>0$ and $C_{q}^{(1)}>0$ such that for fixed $\chi_{1} \in C_{c}^{\infty}\left(B_{\delta_{1}}(\mathcal{C})\right)$ the following holds: For any $A, B \in L^{2 q}\left(\mathbb{R}^{2}, \operatorname{Mat}(2 \times 2 ; \mathbb{C})\right)$ and $z \in \rho\left(D_{\text {trig }}\right)$ we have the inequality

$$
\left\|A \chi_{1}(-\mathrm{i} \nabla)\left(D_{\text {trig }}-z\right)^{-1} B\right\|_{\mathfrak{S}^{\alpha}, 1 / 2,2} \leq C_{q}^{(1)} N_{q}(z)\|A\|_{2 q}\|B\|_{2 q},
$$

where $N_{q}: \mathbb{C} \backslash\{0,1 / 16\} \rightarrow \mathbb{R}_{+}$is a continuous function satisfying

$$
N_{q}(z)= \begin{cases}|z|^{\frac{1}{q}-1} & \text { if }|z| \leq 1 / 64 \\ v(z)|z-1 / 16|^{\frac{1}{q}-1} & \text { if }|z-1 / 16| \leq 1 / 64 .\end{cases}
$$

with $v(z)=1$ or $v(z)=-\ln |z-1 / 16|$ according to whether $q>1$ or $q=1$. Proof. We cut up the support of $\chi_{1}$ by a finite partition of unity $1=\sum_{j} \psi_{j}$ such that the support of each $\psi_{j}$ contains exactly one critical point; this is possible by choosing $\delta_{1}$ sufficiently small. The claim thus follows from Lemma 2.5 and Proposition A.11, together with the identity (2.14). 
Proposition 2.9. Let $1 \leq q \leq 3 / 2$ and $\chi_{2} \in C^{\infty}\left(\mathbb{R}^{d}\right)$. There exist $\delta_{2}>0$ and $C_{q}^{(2)}>0$ such that for any $A, B \in L^{2 q}\left(\mathbb{R}^{2}, \operatorname{Mat}(2 \times 2 ; \mathbb{C})\right)$ we have the inequality

$$
\left\|A \chi_{2}(-\mathrm{i} \nabla)\left(D_{\text {trig }}-z\right)^{-1} B\right\|_{\mathfrak{S}^{\max \left(q, \alpha_{q, 1 / 2,2}\right.}} \leq C_{q}^{(2)}(1+|z|)^{\frac{1}{q}-1}\|A\|_{2 q}\|B\|_{2 q}
$$

uniformly in $z \in \rho\left(D_{\text {trig }}\right)$ provided

$$
|z|+\inf \left\{|\xi|: \xi \in \operatorname{supp} \chi_{2}\right\} \geq 1 / \delta_{2} .
$$

Proof. The proof is similar to that of (2.2). Instead of homogeneity, one uses a rescaling argument such as that in the proof of Proposition A.11. We omit the details.

Proposition 2.10. Let $1 \leq q \leq 5 / 4, \chi_{3} \in C_{c}^{\infty}\left(\mathbb{R}^{d} \backslash \mathcal{C}\right)$, where $\mathcal{C}$ is given by (2.7). Then there exists $C_{q}^{(3)}>0$ such that for any $A, B \in L^{2 q}\left(\mathbb{R}^{2}, \operatorname{Mat}(2 \times\right.$ $2 ; \mathbb{C})$ ) and $z \in \rho\left(D_{\text {trig }}\right)$ we have the inequality

$$
\left\|A \chi_{3}(-\mathrm{i} \nabla)\left(D_{\text {trig }}-z\right)^{-1} B\right\|_{\mathfrak{S}^{\alpha} q, 1 / 4,2} \leq C_{q}^{(3)}(1+|z|)^{-1}\|A\|_{2 q}\|B\|_{2 q} .
$$

Proof. Since $D_{\text {trig }}^{2}=P(-\mathrm{i} \nabla) \mathbf{1}_{2 \times 2}$, with $P$ given by $(1.7)$, we have the identity

$$
\left(D_{\text {trig }}-z\right)^{-1}=\left(D_{\text {trig }}+z\right)\left(P(-\mathrm{i} \nabla)-z^{2}\right)^{-1} .
$$

Since the symbol of $\left(D_{\text {trig }}+z\right)$ is bounded by $\mathcal{O}((1+|z|))$ on the support of $\chi_{3}$ it is sufficient to prove that

$$
\|A m(-\mathrm{i} \nabla) B\|_{\mathfrak{S}^{\alpha_{q}}} \leq C\|A\|_{2 q}\|B\|_{2 q},
$$

where

$$
m(\xi):=\frac{\left(1+|z|^{2}\right) \chi_{3}(\xi)}{P(\xi)-z^{2}}
$$

Define

$$
a:=\max _{\xi \in \operatorname{supp} \chi_{3}} P(\xi)<+\infty .
$$

The estimate is trivial for $|z|^{2}>2 a$ since $m(-\mathrm{i} \nabla)$ is then a smoothing operator with uniform bounds in $z$. Assume now that $|z|^{2} \leq 2 a$, and let $\psi \in C_{c}^{\infty}\left(\mathbb{R}^{2} ;[0,1]\right)$ be such that

$$
\psi \equiv 1 \quad \text { on } \bigcup_{\lambda^{2} \leq 4 a} M_{\lambda}
$$

Then $(1-\psi(-\mathrm{i} \nabla)) m(-\mathrm{i} \nabla)$ is a smoothing operator with uniform bounds in $|z| \leq 2 a$, and the estimate is again trivial for this part. The estimate for the remaining part $\psi(-\mathrm{i} \nabla) m(-\mathrm{i} \nabla)$ follows from Lemma 2.6 and Corollary A.8 with $k=4$. 


\section{Proofs of Theorems 1.1-1.3}

Proof of Theorem 1.1. Let $A:=|V|^{1 / 2}, B:=U|V|^{1 / 2}$ where $U$ is the partial isometry in the polar decomposition of $V$. By Proposition 2.4 and the BirmanSchwinger principle ${ }^{3}$ it follows that for $z \in \sigma_{\mathrm{p}}(D) \backslash \sigma\left(D_{m}\right)$ we have

$$
1 \leq\left\|A\left(D_{m}-z\right)^{-1} B\right\| \leq C|k(z)|^{\frac{2}{q}-2}\left(|\zeta(z)|+|\zeta(z)|^{-1}\right)\|V\|_{q} .
$$

This immediately yields (1.4). ${ }^{4}$ The case $z \in \sigma_{\mathrm{p}}(D)$ follows from [10, Proposition 3.1]. One obtains (1.5) from (1.4) by solving for $|\zeta(z)|$ and Taylor expanding in $\|V\|_{1}$. This kind of computation may be found in the proof of Theorem $2.1 \mathrm{in}$ [4] and is therefore omitted. The claim $m=0$ is obvious since $|\zeta(z)|=1$ in this case.

Proof of Theorem 1.3. Let $\mathcal{C}$ be the set of critical points of $P$, see (2.7). Let $C_{q}^{(1)}, C_{q}^{(2)}, C_{q}^{(3)}, \delta_{1}, \delta_{2}$ be as in Propositions 2.8-2.10 and set $\delta:=\min \left(\delta_{1}, \delta_{2}\right)$. Let $z \in \sigma_{\mathrm{p}}(D) \cap \rho\left(D_{\text {trig }}\right)$. Proposition 2.9 with $\chi_{2} \equiv 1$ and the BirmanSchwinger principle imply that either $|z| \leq \delta^{-1}$ or that $1 \leq C_{q}^{(2)}|z|^{\frac{1}{q}-1}\|V\|_{q}$. Hence,

$$
|z|^{q-1} \leq \max \left(\delta^{1-q},\left(C_{q}^{(2)}\right)^{q}\|V\|_{q}^{q}\right),
$$

proving (1.8). To prove (1.9), we may assume that

$$
\operatorname{dist}(z,\{0,1 / 16\}) \leq \min \left(1 / 64,\left(C_{q}^{(1)} / C_{q}^{(2)}\right)^{q /(q-1)}\right),
$$

otherwise the claim follows from (1.8). Let $1=\chi_{1}+\chi_{2}+\chi_{3}$ be a partition of unity subordinate to the decomposition

$$
\mathbb{R}^{2}=B_{\delta}(\mathcal{C}) \cup\left(\mathbb{R}^{2} \backslash B_{1 / \delta}(0)\right) \cup\left(B_{1 / \delta}(0) \backslash B_{\delta}(\mathcal{C})\right) .
$$

The Birman-Schwinger principle together with Propositions 2.8-2.10 yields

$$
1 \leq \sum_{j=1}^{3}\left\|A\left(D_{\text {trig }}-z\right)^{-1} B\right\| \leq\left(C_{q}^{(1)} N_{q}(z)+C_{q}^{(2)}\right)\|V\|_{q}+C_{q}^{(3)} c_{1},
$$

where $N_{q}(z)$ is defined in (2.11). By (3.1) we have $N_{q}(z) \geq C_{q}^{(2)} / C_{q}^{(1)}$, hence, if $c_{1}<\left(C_{q}^{(3)}\right)^{-1}$, then

$$
N_{q}(z)^{-q} \leq\left(\frac{2 C_{q}^{(2)}}{1-C_{q}^{(3)} c_{1}}\right)^{q}\|V\|_{q}^{q},
$$

which implies (1.9). If $q=1$, one has to multiply $N_{q}(z)$ by $-\ln |z-1 / 16|$ in (3.2) to obtain (1.10) analogously.

\footnotetext{
${ }^{3}$ The operators considered here fit into the general framework of [11], for example. We refer to that paper and to [4] for a discussion of the operator theoretic aspects, including the Birman-Schwinger principle.

${ }^{4}$ Strictly speaking, we have only proved (1.4) for $z \notin \sigma\left(D_{0}\right)$. However, embedded eigenvalues can be included by [10, Proposition 3.1]. This applies to the proof of Theorem 1.3 as well.
} 


\section{Appendix A. Frequency-localized resolvent estimates in $d \geq 2$}

Definition A.1. Let $S \subset \mathbb{R}^{d}$ be a smooth hypersurface. A defining function of $S$ is a smooth function $\rho: \mathbb{R}^{d} \rightarrow \mathbb{R}$ such that $S=\left\{\xi \in \mathbb{R}^{d}: \rho(\xi)=0\right\}$ and $|\nabla \rho|>0$ on $S$. If in addition $|\nabla \rho|=1$ on $S$, we call $\rho$ a normalized defining function of $S$.

Definition A.2. Let $S \subset \mathbb{R}^{d}$ be a smooth hypersurface with normalized defining function $\rho: \mathbb{R}^{d} \rightarrow \mathbb{R}$. The second fundamental form of $S$ at $\xi_{0} \in S$ is the restriction of $\nabla^{2} \rho\left(\xi_{0}\right)$ to the tangent space $T_{\xi_{0}} S \subset \mathbb{R}^{d}$, and its eigenvalues are the principal curvatures at $\xi_{0}$. The Gaussian curvature is the product of all principal curvatures.

Definition A.3. A smooth hypersurface $S \subset \mathbb{R}^{d}$ is called of finite type at $\xi_{0} \in S$ if at least one of its principal curvatures does not vanish to infinite order at $\xi_{0}$.

Remark A.4. In analogy to Remark 2.2, if $S$ is locally the graph of a smooth function $h: U \rightarrow \mathbb{R}$ defined near the origin and with $h(0)=0, \nabla h(0)=0$, then the type $k$ of $S$ at the origin equals the smallest $k \in \mathbb{N}_{>2}$ such that $\partial^{\alpha} h(0) \neq 0$ for some $\alpha \in \mathbb{N}_{0}^{d-1}$ of length $|\alpha| \leq k$, see [21, VII.3.2].

Proposition A.5. Let $S \subset \mathbb{R}^{d}$ be a smooth hypersurface with normalized defining function $\rho: \mathbb{R}^{d} \rightarrow \mathbb{R}$. Fix $\chi \in C^{\infty}\left(\mathbb{R}^{d}\right)$ and assume that

$$
\sup _{x \in \mathbb{R}^{d}}(1+|x|)^{r}\left|\widehat{\chi \mathrm{d} \sigma_{S}}(x)\right|<\infty
$$

for some $r>0$, where $\mathrm{d} \sigma_{S}$ is the canonical surface measure on $S$. Let $1 \leq$ $q \leq 1+r$ and define

$$
\alpha_{q, r, d}:= \begin{cases}\frac{2(d-1-r) q}{d-q}, & \text { if } \frac{d}{d-r} \leq q \leq 1+r, \\ \frac{2 r q+}{2 r q-d(q-1)}, & \text { if } 1 \leq q<\frac{d}{d-r} .\end{cases}
$$

Here, $2 r q+$ means $2 r q+\varepsilon$ with $\varepsilon>0$ arbitrarily small but fixed. Then for all $A, B \in L^{2 q}\left(\mathbb{R}^{2}\right)$ and all $z \in \mathbb{C} \backslash \mathbb{R}$ we have the estimate

$$
\left\|A \chi(-\mathrm{i} \nabla)(\rho(-\mathrm{i} \nabla)-z)^{-1} B\right\|_{\mathfrak{S}^{\alpha}, r, d} \leq C\|A\|_{2 q}\|B\|_{2 q},
$$

with a constant $C$ independent of $z, A, B$.

The proof relies on the following lemma.

Lemma A.6 (pointwise bounds on complex powers). Let $h: \mathbb{R}^{n} \rightarrow \mathbb{R}$ be a smooth real-valued function and fix $\psi \in C^{\infty}\left(\mathbb{R}^{n}\right)$. Assume that

$$
\int_{\mathbb{R}^{n}} \mathrm{e}^{\mathrm{i} x h(\eta)} \psi(\eta) \mathrm{d} \eta=\mathcal{O}\left((1+|x|)^{-r}\right), \quad x \in \mathbb{R}
$$

for some $r>0$. Given $a \in[1,1+r], t \in \mathbb{R}$, define the tempered distributions

$$
u_{a, t}^{ \pm}(\xi, \eta)=\mathrm{e}^{\pi^{2}(a+\mathrm{i} t)^{2}} \psi(\eta)(\xi-h(\eta) \pm \mathrm{i} 0)^{-a-\mathrm{i} t}, \quad \xi \in \mathbb{R}, \quad \eta \in \mathbb{R}^{n} .
$$




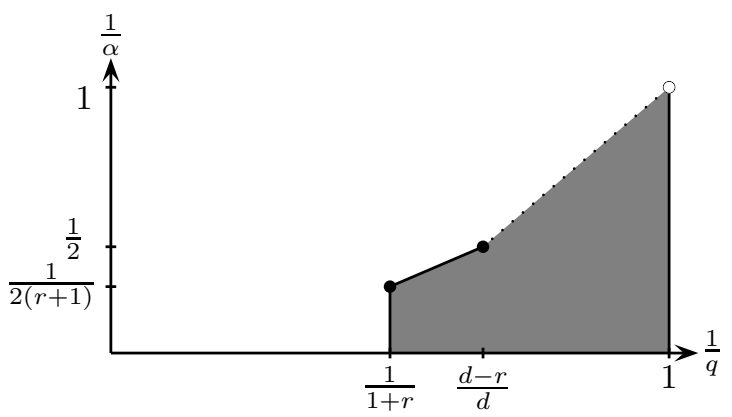

FiguRE 1. Range of validity of the estimate (A.3).

Then the $(n+1)$-dimensional inverse Fourier transform $v_{a, t}^{ \pm}=\mathcal{F}^{-1} u_{a, t}^{ \pm}$satisfies the pointwise estimate

$$
\sup _{t \in \mathbb{R}} \sup _{(x, y) \in \mathbb{R} \times \mathbb{R}^{n}}(1+|x|+|y|)^{1+r-a}\left|v_{a, t}^{ \pm}(x, y)\right|<\infty .
$$

Proof. By a change of variables $\tau=\xi-h(\eta)$ and by [13, Example 7.1.17] the partial inverse Fourier transform of $(\xi-h(\eta) \pm \mathrm{i} 0)^{-a-\mathrm{i} t}$ with respect to the variable $\tau$ is given by

$$
\mathcal{F}_{\tau \rightarrow x}^{-1}\left\{(\tau-h(\eta) \pm \mathrm{i} 0)^{-a-\mathrm{i} t}\right\}(x)=(2 \pi)^{-1 / 2} \mathrm{e}^{ \pm \mathrm{i} \pi(a+\mathrm{i} t) / 2} \mathrm{e}^{\mathrm{i} x h(\eta)} \chi_{\mp}^{a+\mathrm{i} t-1}(x)
$$

where $\chi_{ \pm}^{z}$ are distributions on $\mathbb{R}$ given by

$$
\chi_{ \pm}^{z}(x)=\frac{x_{ \pm}^{z}}{\Gamma(z+1)}, \quad z \in \mathbb{C} .
$$

Observing that $|\Gamma(a+\mathrm{i} t)|^{-1} \leq C \mathrm{e}^{\pi^{2}|a+\mathrm{i} t|^{2} / 2}$, we get the desired bound by combining (A.4) and (A.6).

Proof of Proposition A.5. The proof is similar to that of Lemma 4.4 in [2]. The difference is that the pointwise estimate (4.13) in [2] is replaced by

$$
\sup _{x \in \mathbb{R}^{d}}(1+|x|)^{1+r-a}\left|\chi(-\mathrm{i} \nabla)(\rho(-\mathrm{i} \nabla)-z)^{-(a+\mathrm{i} t)}(x)\right| \leq C \mathrm{e}^{C t^{2}},
$$

where $1 \leq a \leq 1+r$ and $t \in \mathbb{R}$. The above is a consequence of (A.1) and Lemma A. 6 with $n=d-1$. This can be seen by invoking the implicit function theorem, by which we may locally write

$$
\rho(\xi)=e(\xi)\left(\xi_{1}-h\left(\xi^{\prime}\right)\right), \quad \xi=\left(\xi_{1}, \xi^{\prime}\right),
$$

in appropriate coordinates, where $e \neq 0$. Then $S$ is locally the graph of $h$ over the $\xi^{\prime}$-plane and (A.4) is satisfied. Together with the Hardy-LittlewoodSobolev inequality, (A.7) implies the Hilbert-Schmidt bound

$$
\left\|A^{a+\mathrm{i} t} \chi(-\mathrm{i} \nabla)(\rho(-\mathrm{i} \nabla)-z)^{-(a+\mathrm{i} t)} B^{a+\mathrm{i} t}\right\|_{\mathfrak{S}^{2}}^{2} \leq C \mathrm{e}^{C t^{2}}\|A\|_{\frac{2 a d}{d-1-r+a}}\|B\|_{\frac{2 a d}{d-1-r+a}}
$$


for $1 \leq a \leq 1+r$. Complex interpolation with the trivial bound

$$
\left\|A^{\mathrm{i} t} \chi(-\mathrm{i} \nabla)(\rho(-\mathrm{i} \nabla)-z)^{-\mathrm{i} t} B^{\mathrm{i} t}\right\|_{\mathfrak{S}^{2}}^{2} \leq C \mathrm{e}^{C t^{2}}
$$

yields (A.3) for $\frac{d}{d-r} \leq q \leq 1+r$. The estimate for $1 \leq q<\frac{d}{d-r}$ does not depend on the assumption (A.1); the argument is similar to the case $1 \leq q<\frac{2 d}{d+1}$ in the proof of [2, Lemma 4.4]. As in that case one proves

$$
\left\|A \chi(-\mathrm{i} \nabla)(\rho(-\mathrm{i} \nabla)-z)^{-(b+\mathrm{i} t)} B\right\|_{\mathfrak{S}^{1}} \leq C \mathrm{e}^{C t^{2}}(1-b)^{-1}\|A\|_{2}\|B\|_{2}, \quad 0<b<1
$$

and interpolates with

$$
\left\|A \chi(-\mathrm{i} \nabla)(\rho(-\mathrm{i} \nabla)-z)^{-(1+r+\mathrm{i} t)} B\right\|_{\mathfrak{S}^{2}} \leq C \mathrm{e}^{C t^{2}}\|A\|_{2}\|B\|_{2},
$$

giving

$$
\left\|A \chi(-\mathrm{i} \nabla)(\rho(-\mathrm{i} \nabla)-z)^{-1} B\right\|_{\mathfrak{S}^{1+}} \leq C\|A\|_{2}\|B\|_{2} .
$$

The last bound is again interpolated with the estimate (A.3) for $q$ in the range already proven. This yields (A.3) for the whole range of $q$; see Figure A.

Remark A.7. In [2, Lemma 4.4] we have $\rho(\xi):=T(\xi)-\lambda$, and the estimate was proved under the assumption that $S$ has everywhere nonvanishing Gaussian curvature. The latter implies that (A.1) holds with $r=(d-1) / 2$. The Schatten space estimates for the resolvent of the Laplacian were first proved by Frank-Sabin [9]. By a duality argument, (A.3) implies

$$
\left\|\chi(-\mathrm{i} \nabla)(\rho(-\mathrm{i} \nabla)-z)^{-1}\right\|_{L^{p} \rightarrow L^{p^{\prime}}} \leq C, \quad \frac{1}{r+1} \leq \frac{1}{p}-\frac{1}{p^{\prime}} \leq 1,
$$

but is strictly stronger; in fact, (A.9) is equivalent to (A.3) with $\alpha_{q, r, d}=\infty$. The estimate (A.9) for the imaginary part of the resolvent follows already from a result of Greenleaf [12, Theorem 1]; see also [21, VII.5.15].

Corollary A.8. Assume that $S \cap \operatorname{supp} \chi$ is compact and of finite type $k$. Then (A.3) holds with $r=1 / k$.

Proof. Under the finite type assumption (A.1) holds with $r=1 / k$. For $d=$ 2 this follows from Lemma 2.3. For $d \geq 3$ it follows from [21, Theorem VIII.2].

Corollary A.9. Assume that $S \cap \operatorname{supp} \chi$ is compact and has at least $l \leq d-1$ everywhere nonvanishing principal curvatures. Then (A.3) holds with $r=l / 2$. The constant is locally uniform in $\rho$ in the $C^{2}$-topology.

Proof. Littman [18] proved that the stated assumption implies the decay estimate (A.1) with $r=l / 2$. The last claim follows from Lemma A.10.

Lemma A.10 (Stability of curvature). Let $S_{1}$ and $S_{2}$ be smooth hypersurfaces in $\mathbb{R}^{d}$ with normalized defining functions $\rho_{1}$ and $\rho_{2}$. Assume that $S_{1}$ has $l \leq d-1$ nonvanishing principal curvatures at $\xi_{0} \in S_{1}$. Then there exists a neighborhood $U$ of $\xi_{0}$ in $\mathbb{R}^{d}$ and a constant $c>0$ such that whenever $\left\|\rho_{1}-\rho_{2}\right\|_{C^{2}(U)} \leq c$, then $S_{2}$ has l nonvanishing principal curvatures in $S_{2} \cap U$. 
Proof. By assumption, we have

$$
\sup _{\xi \in U}\left\|\nabla^{2} \rho_{1}-\nabla^{2} \rho_{2}\right\| \leq d^{1 / 2} c,
$$

where $\|\cdot\|$ denotes the operator norm of $d \times d$-matrices. Let $U$ be a neighborhood of $\xi_{0}$ in $\mathbb{R}^{d}$ such that $\left\|\rho_{1}-\rho_{2}\right\|_{C^{2}(U)} \leq c$. We will later choose $\operatorname{diam}(U)$ and $c>0$ sufficiently small. Let $\xi_{0} \in S_{1}$ and $\eta_{0} \in S_{2} \cap U$. Using the triangle inequality, we get

$$
\left|\nabla \rho_{1}\left(\xi_{0}\right)-\nabla \rho_{2}\left(\eta_{0}\right)\right| \leq c+\left\|\rho_{2}\right\|_{C^{2}(U)} \operatorname{diam}(U) .
$$

Let $P_{1}$ and $P_{2}$ be the orthogonal projections in $\mathbb{R}^{d}$ onto the tangent spaces $T_{\xi_{0}} S_{1}=\left\{\nabla \rho_{1}\left(\xi_{0}\right)\right\}^{\perp}$ and $T_{\eta_{0}} S_{2}=\left\{\nabla \rho_{2}\left(\eta_{0}\right)\right\}^{\perp}$, respectively. Then by (A.11),

$$
\left\|P_{1}-P_{2}\right\|^{2}=1-\left\langle\nabla \rho_{1}\left(\xi_{0}\right), \nabla \rho_{2}\left(\eta_{0}\right)\right\rangle_{\mathbb{R}^{d}} \leq c+\left\|\rho_{2}\right\|_{C^{2}(U)} \operatorname{diam}(U) .
$$

Set $A_{1}:=\nabla^{2} \rho_{1}\left(\xi_{0}\right)$ and $A_{2}:=\nabla^{2} \rho_{2}\left(\eta_{0}\right)$. Then (A.10) and (A.12) imply that

$$
\begin{aligned}
\left\|P_{1} A_{1} P_{1}-P_{2} A_{2} P_{2}\right\| & =\left\|P_{2}\left(A_{1}-A_{2}\right) P_{2}+P_{2} A_{1}\left(P_{1}-P_{2}\right)+\left(P_{1}-P_{2}\right) A_{1} P_{1}\right\| \\
& \leq\left\|A_{1}-A_{2}\right\|+2\left\|A_{1}\right\|\left\|P_{1}-P_{2}\right\| \\
& \leq d^{1 / 2} c+2\left\|\rho_{1}\right\|_{C^{2}(U)}\left(c+\left\|\rho_{2}\right\|_{C^{2}(U)} \operatorname{diam}(U)\right)^{1 / 2} .
\end{aligned}
$$

By assumption, the spectrum of $P_{1} A_{1} P_{1}$ contains at least $l$ nonzero eigenvalues $\lambda_{1}, \ldots, \lambda_{k}$. Define $\varepsilon:=\min _{1 \leq j \leq k}\left|\lambda_{j}\right|>0$, and choose $U, c$ such that

$$
d^{1 / 2} c+2\left\|\rho_{1}\right\|_{C^{2}(U)}\left(c+\left\|\rho_{2}\right\|_{C^{2}(U)} \operatorname{diam}(U)\right)^{1 / 2} \leq \varepsilon / 2 .
$$

By the stability of bounded invertibility it follows that $P_{2} A_{2} P_{2}$ has at least $l$ nonzero eigenvalues. This proves the claim. point.

The following is a version of [2, Lemma 4.4] near a nondegenerate critical

Proposition A.11. Let $T: \mathbb{R}^{d} \rightarrow \mathbb{R}$ be a smooth function with a nondegenerate critical point $\xi_{0}$ and corresponding value $\lambda_{\mathrm{c}} \in \mathbb{R}$. Then there exists $\delta>0$ such that for fixed $\chi \in C_{c}^{\infty}\left(B_{\delta}\left(\xi_{0}\right)\right), d / 2<q \leq(d+1) / 2$ and for all $A, B \in$ $L^{2 q}\left(\mathbb{R}^{2}\right), z \in \rho(T(-\mathrm{i} \nabla))$, we have the estimate

$$
\left\|A \chi(-\mathrm{i} \nabla)(T(-\mathrm{i} \nabla)-z)^{-1} B\right\|_{\mathfrak{S}^{\alpha}, r, d} \leq C\left|z-\lambda_{\mathrm{c}}\right|^{\frac{d}{2 q}-1}\|A\|_{2 q}\|B\|_{2 q},
$$

uniformly for $z$ in a punctured neighborhood of $\lambda_{c}$. Here,

$$
\alpha_{q, r, d}:= \begin{cases}\frac{q(d-1)}{d-q}, & \text { if } \frac{2 d}{d+1} \leq q \leq \frac{d+1}{2}, \\ \frac{q(d-1)+}{d-q}, & \text { if } 1 \leq q<\frac{2 d}{d+1} .\end{cases}
$$

If $\xi_{0}$ is a local extremum, then the same estimate holds for $q=d / 2$. If $\xi_{0}$ is a saddle point the same is true if $C$ is replaced by $-C \ln \left|z-\lambda_{\mathrm{c}}\right|$.

Proof. Without loss of generality we may assume that $\xi_{0}=0$ and $\lambda_{c}=0$. We will pick $\delta$ at least so small that $B_{\delta}(0)$ does not contain any other critical point besides the origin. Let $Q$ be the quadratic form

$$
Q(\xi):=\frac{1}{2}\langle\xi, H \xi\rangle_{\mathbb{R}^{d}}
$$


where $H=\nabla^{2} T(0)$ is the Hessian of $T$ at the origin. By a linear change of variables we may assume that

$$
Q(\xi)=\xi_{1}^{2}+\ldots+\xi_{j}^{2}-\xi_{j+1}^{2}-\xi_{d}^{2}
$$

where $2 j-d$ is the signature of $Q$. By standard arguments involving the Phragmen-Lindelöf maximum principle we may assume that $z=\lambda \pm \mathrm{i} 0$, where $\lambda \in \mathbb{R}$ lies in a small punctured neighborhood of the origin in $\mathbb{R}$. We will not need to distinguish between the two limits and so, by abuse of notation, we just write $\lambda$ to denote either of those limits. Moreover, by possibly multiplying $T$ by -1 we may assume $\lambda>0$. By a change of scale $\xi=\lambda^{1 / 2} \eta$, it then suffices to prove that

$$
\left\|A \chi(-\mathrm{i} \nabla)\left(\lambda^{-1} T\left(-\mathrm{i} \lambda^{1 / 2} \nabla\right)-1\right)^{-1} B\right\|_{\mathfrak{S}^{\alpha q, r, d}} \leq C\|A\|_{2 q}\|B\|_{2 q},
$$

where $\chi$ is now supported in $B_{\delta \lambda^{-1 / 2}}(0)$. We have

$$
\lambda^{-1} T\left(\lambda^{1 / 2} \eta\right)-1=Q(\eta)-1+\lambda^{1 / 2} \mathcal{O}_{C^{n}}\left(|\eta|^{3}\right), \quad \eta \in B_{\delta \lambda^{-1 / 2}}(0)
$$

for any fixed $n$, which we assume to be sufficiently large. Here, $g=\mathcal{O}_{C^{n}}\left(|\xi|^{3}\right)$ means $\partial^{\alpha} g=\mathcal{O}\left(|\xi|^{(3-|\alpha|)+}\right)$ for $|\alpha| \leq n$ as $\xi \rightarrow 0$. If we set $M_{\lambda}:=\left\{\xi \in \mathbb{R}^{d}\right.$ : $T(\xi)=\lambda\}$, then $\rho(\eta):=\lambda^{-1} T\left(\lambda^{1 / 2} \eta\right)-1$ is an approximately normalized defining function of $S:=\lambda^{-1 / 2} M_{\lambda}$ in the sense that $c \leq|\nabla \rho| \leq 1 / c$ on $S$ with a constant $c>0$ that is uniform for $\lambda>0$ in a small neighborhood of the origin; hence we may as well assume $\rho$ to be normalized.

We first consider the cases when the signature of $Q$ is $d$ or $-d$. Since these two cases differ from each other only by a change of sign we only treat the first one. Since $|\rho(\eta)| \geq 1 / 2$ for $|\eta| \leq 1 / 2$ or $|\eta| \geq 3 / 2$ and for $\delta$ sufficiently small, we may restrict our attention to the region $A:=\left\{\eta \in \mathbb{R}^{d}: 1 / 2 \leq|\eta| \leq\right.$ $3 / 2\}$, i.e. we assume that $\chi$ localizes to this region. In view of (A.16), $\rho$ is an $\mathcal{O}_{C^{n}(A)}\left(\lambda^{1 / 2}\right)$-perturbation of $\rho_{1}(\eta):=Q(\eta)-1$. Since $\rho_{1}$ is an approximately normalized defining function of the unit sphere we can apply Corollary A.9 to conclude (A.15).

Now consider the case when the signature of $Q$ is different from $\pm d$, i.e. when $j \in\{1, \ldots, d-1\}$ in (A.14). The set $\left\{\eta \in \mathbb{R}^{d}: Q(\eta)=1\right\}$ is a two-sheeted hyperboloid, which in contrast to the sphere in the previous case is noncompact. While the localization $\chi$ introduces a cutoff to the frequency scale $|\eta| \leq \delta \lambda^{-1 / 2}$, the latter is not uniform with respect to small $\lambda$, so we cannot apply Corollary A.9. Instead, we will prove that a modification of (A.4) holds and appeal to (the proof of) Proposition A.5 directly. By the implicit function theorem we may solve the equation $\rho(\eta)=0$ for one of the first $j$ variables. By a partition of unity of the $j-1$ sphere we may thus assume that $\rho(\eta)=e(\eta)\left(\eta_{1}-h\left(\eta^{\prime}\right)\right)$ on the set where $|\rho(\eta)| \leq 1 / 2$. Recalling that $\lambda$ is a small positive number, let $1=\sum_{k=0}^{-c \ln \lambda} \phi_{k}\left(\eta^{\prime}\right)$ be a Littlewood-Paley decomposition of the set $\left|\eta^{\prime}\right| \leq \delta \lambda^{-1 / 2}$. We claim that

$$
\left.\left|\mathcal{F}^{-1}\left\{\phi_{k}(\rho \pm \mathrm{i} 0)^{-a-\mathrm{i} t}\right\}(x)\right| \lesssim 2^{k(d-2 a)}\left(1+2^{k}|x|\right)\right)^{-\frac{d-1}{2}}\left|2^{k} x\right|^{a-1} .
$$


By a change of variables and in view of the obvious lower bound $|e(\eta)| \gtrsim|\eta|$ the proof would follow from the pointwise estimate

$$
\int_{\mathbb{R}^{d-1}} \mathrm{e}^{\mathrm{i} x h\left(2^{k} \eta^{\prime}\right)} \psi\left(\eta^{\prime}\right) \mathrm{d} \eta^{\prime}=\mathcal{O}\left(\left(1+2^{k}|x|\right)\right)^{-\frac{d-1}{2}}, \quad x \in \mathbb{R}
$$

analogously to the proof of Lemma A.6. Summing (A.17) over $0 \leq k \leq-c \ln \lambda$ we get

$$
\mathcal{F}^{-1}\left\{\chi(\rho \pm \mathrm{i} 0)^{-a-\mathrm{i} t}\right\} \in L^{\infty}\left(\mathbb{R}^{d}\right), \quad \frac{d}{2}<a \leq \frac{d+1}{2},
$$

locally uniformly in $\lambda$. At the endpoint $a=d / 2$ one obtains an $\mathcal{O}(-\ln \lambda)$ bound due to logarithmic divergence of the sum. The proof of (A.18) follows by standard stationary phase arguments upon observing that the Hessian of $2^{-k} h\left(2^{k} \eta^{\prime}\right)$ is uniformly nondegenerate; this may be seen by differentiating the implicit equation $\rho\left(h\left(\eta^{\prime}\right), \eta^{\prime}\right)=0$ twice.

Acknowledgements. The author thanks the referees for their comments.

\section{References}

[1] J. Aguilar and J. M. Combes. A class of analytic perturbations for one-body Schrödinger Hamiltonians. Comm. Math. Phys., 22:269-279, 1971.

[2] J.-C. Cuenin. Eigenvalue bounds for Dirac and fractional Schrödinger operators with complex potentials. J. Funct. Anal., 272(7):2987-3018, 2017.

[3] J.-C. Cuenin. Embedded eigenvalues for generalized Schrödinger operators. ArXiv:1709.06989, page 18 pages, 2017.

[4] J.-C. Cuenin, A. Laptev, and C. Tretter. Eigenvalue estimates for nonselfadjoint Dirac operators on the real line. Ann. Henri Poincaré, 15(4):707$736,2014$.

[5] J.-C. Cuenin and P. Siegl. Eigenvalues of one-dimensional non-self-adjoint dirac operators and applications. Letters in Mathematical Physics, pages 1-22, Jan 2018.

[6] F. Ferrulli, A. Laptev, and O. Safronov. Eigenvalues of the bilayer graphene operator with a complex valued potential. ArXiv e-prints, Dec. 2016.

[7] R. L. Frank. Eigenvalue bounds for Schrödinger operators with complex potentials. Bull. Lond. Math. Soc., 43(4):745-750, 2011.

[8] R. L. Frank. Eigenvalue bounds for Schrödinger operators with complex potentials. III. Trans. Amer. Math. Soc., 370(1):219-240, 2018.

[9] R. L. Frank and J. Sabin. Restriction theorems for orthonormal functions, Strichartz inequalities, and uniform Sobolev estimates. Amer. J. Math., 139(6):1649-1691, 2017.

[10] R. L. Frank and B. Simon. Eigenvalue bounds for Schrödinger operators with complex potentials. II. J. Spectr. Theory, 7(3):633-658, 2017.

[11] F. Gesztesy, Y. Latushkin, M. Mitrea, and M. Zinchenko. Nonselfadjoint operators, infinite determinants, and some applications. Russ. J. Math. Phys., 12(4):443-471, 2005. 
[12] A. Greenleaf. Principal curvature and harmonic analysis. Indiana Univ. Math. J., 30(4):519-537, 1981.

[13] L. Hörmander. The analysis of linear partial differential operators. I, volume 256 of Grundlehren der Mathematischen Wissenschaften [Fundamental Principles of Mathematical Sciences]. Springer-Verlag, Berlin, second edition, 1990. Distribution theory and Fourier analysis.

[14] T. Kato. Wave operators and similarity for some non-selfadjoint operators. Math. Ann., 162:258-279, 1965/1966.

[15] M. I. Katsnelson. Graphene: Carbon in Two Dimensions. Cambridge University Press, 2012.

[16] C. E. Kenig, A. Ruiz, and C. D. Sogge. Uniform Sobolev inequalities and unique continuation for second order constant coefficient differential operators. Duke Math. J., 55(2):329-347, 1987.

[17] A. Laptev and O. Safronov. Eigenvalue estimates for Schrödinger operators with complex potentials. Comm. Math. Phys., 292(1):29-54, 2009.

[18] W. Littman. Fourier transforms of surface-carried measures and differentiability of surface averages. Bull. Amer. Math. Soc., 69:766-770, 1963.

[19] U. V. Riss and H.-D. Meyer. Calculation of resonance energies and widths using the complex absorbing potential method. J. Phys. B, 26(23):4503-4535, 1993.

[20] B. Simon. Trace ideals and their applications, volume 120 of Mathematical Surveys and Monographs. American Mathematical Society, Providence, RI, second edition, 2005.

[21] E. M. Stein. Harmonic analysis: real-variable methods, orthogonality, and oscillatory integrals, volume 43 of Princeton Mathematical Series. Princeton University Press, Princeton, NJ, 1993. With the assistance of Timothy S. Murphy, Monographs in Harmonic Analysis, III.

[22] M. A. Vozmediano, M. Katsnelson, and F. Guinea. Gauge fields in graphene. Physics Reports, 496(4):109-148, 2010.

[23] M. Zworski. Scattering resonances as viscosity limits. ArXiv e-prints 1505.00721, May 2015.

Jean-Claude Cuenin

Mathematisches Institut, Ludwig-Maximilians-Universität München, $80333 \mathrm{Mu}$ nich, Germany

e-mail: cuenin@math. $1 \mathrm{mu} . \mathrm{de}$ 